\title{
Aeroservoelastic modelling and control of a slender anti-air missile for active damping of longitudinal bending vibrations
}

\author{
Anatole Verhaegen ${ }^{\mathrm{a}}$, Rafał Żbikowskib,* \\ ${ }^{a}$ Airbus Defence and Space SAS, 31 rue des Cosmonautes, Z.l. du Palays 31402, Toulouse Cedex 4, FRANCE \\ ${ }^{b}$ School of Aerospace, Transport and Manufacturing, Cranfield University, Cranfield, Bedfordshire MK43 OAL, ENGLAND
}

\begin{abstract}
Slender anti-air missiles experience longitudinal bending in supersonic flight and yet their autopilots are designed under the rigid-body assumption. Such autopilot design employs notch filters to remove the modal frequencies of the elastic airframe but this approach limits the autopilot bandwidth. In this paper, aeroservoelastic modelling and control of the ASTER 30 missile is proposed to enable autopilot design with extended bandwidth.

The aeroservoelastic model combines missile flight dynamics, actuator dynamics and airframe elasticity, the latter focusing on longitudinal bending treated as a continuous Euler-Bernoulli beam problem. The beam is discretised leading to a nodal model and the modal analysis is then performed. The modal model is expressed in the state-space form and its order is reduced to enable optimal sensor placement and active damping control. The aeroservoelastic model of the ASTER 30 missile is further refined for control purposes by optimally choosing actuator inputs together with the number and position of sensors to be mounted on the missile airframe. Once these choices are made, several variants of active vibration damping control are proposed and analysed in order to enable an extended bandwidth for the autopilot by countering the airframe deformation measured by these sensors.
\end{abstract}

Keywords:

flexible missile, aeroservoelastic model, bending vibrations, optimal sensor placement, active vibration damping

\section{Introduction}

This work is motivated by the need to improve flight control performance of agile missiles in the context of modern air defence systems. Bending effects for fast, slender anti-air missiles are pronounced and may be misinterpreted by on-board sensors as deviations from the rigid-body trajectory and lead to parasitic actuation, wasting energy and affecting stability. Autopilots for such missiles are designed under the rigid-body assumptions despite longitudinal bending of the missile airframe. In the corresponding missile autopilot design, notch filters are used to remove the modal frequencies of the elastic airframe but this approach limits the autopilot bandwidth and does not counter the airframe deformation. An alternative approach, not much exploited in the missile context, is to use active vibration damping which: 1) minimises the airframe deformation, 2)

\footnotetext{
* Corresponding author

Email address: r.w.zbikowski@cranfield.ac.uk (Rafał Żbikowski)
}

Preprint submitted to Aerospace Science and Technology extends the autopilot bandwidth, and 3) allows designing less stiff (and thus lighter) airframes [1]. A starting point for this alternative approach is the development of a tractable aeroservoelastic model of the missile to serve two purposes: 1) to capture the dominant effects of longitudinal bending vibrations, and 2) to design active damping of these vibrations in order to dispense with the notch filter and extend the autopilot bandwidth.

Literature on flexible missile modelling is limited and includes the case of a spinning aiframe [2]. In [3], continuing the work of [2], a basic aeroelastic model was proposed for a spinning missile represented by three rigid bodies linked with massless beams. The bending mode frequencies were within $5 \%$ the real frequencies but the mode shapes were rather inaccurate making this approach unattractive for sensor placement. A more complex model was investigated in [4] by considering continuous bending and torsion but the complexity of this model makes it impractical for sensor placement and control design. A planar model of a flexible missile was derived in [5] and considered only yaw, leading to considerable complexity because of a discrete flex-

17th February 2017

Published by Elsevier. This is the Author Accepted Manuscript issued with: 
ible hinge in the middle of the body, used to express elasticity of the link between the two stages.

In this paper, a tractable aeroservoeleastic model for the ASTER 30 missile is outlined in Section 2 and then optimal actuator and sensor selection is addressed. After noting in Section 3 that adding actuators to an existing missile is not a realistic option, the focus shifts to optimal sensor placement, presented in Section 4 A combination of the existing actuators and additional (optimally-placed) sensors enables design of several active vibration damping controllers, see Section 5. Finally, conclusions are given in Section 6 .

\section{ASTER-30 aeroservoeleastic model}

In this paper, the reference platform is the two-stage anti-air ASTER 30 missile which has a dart stacked on top of a booster, see Figure 2. During the acceleration phase, the booster uses thrust vectoring and after separation, a two-actuator system called PIF PAF takes over but the focus here is on the missile flight before separation. At launch, ASTER 30 has the total mass of $m=450 \mathrm{~kg}$, rotational inertia of $J_{y}=789 \mathrm{~kg} \cdot \mathrm{m}^{2}$, the total length of $L=4.9 \mathrm{~m}$ and the centre of gravity (CG) position at $x_{C G}=1.86 \mathrm{~m}$; the $\mathrm{CG}$ position is assumed to be fixed during the unseparated phase of flight. ASTER 30 is similar to the flared frame studied in [6] so the relevant aerodynamic data from [6] were adopted here.

An aeroservoelastic model of ASTER 30 was derived by combining three parts: 1) linearised flight dynamics for a sea-level, Mach 2 flight at trim in the vertical plane; 2) actuator (servo) dynamics which comprises thrust vectoring and aerodynamic fins, each modelled as a second-order transfer function with cut-off frequencies of $25 \mathrm{~Hz}$ and $50 \mathrm{~Hz}$, respectively, and a damping ratio of $0.7 ; 3$ ) structure dynamics. The structure dynamics were modelled by first discretising a continuous Euler-Bernoulli beam with nodal coordinates [7, Chapter 4] defined through displacements and velocities at selected structural locations (nodes). The nodal model was derived under the initial assumption, revised in Section 4, that forces were applied at all $n=100$ uniformly spaced nodes and that measurements of displacements, rotation rates and linear accelerations at all these nodes were possible. Based on that nodal model, modal coordinates were introduced by considering the displacements and velocities of $n-2$ natural modes.

The modal model resulting from nodal discretisation of the continuous Euler-Bernoulli beam is:

$$
\begin{aligned}
\dot{x} & =A x+B u \\
y & =C x+D u
\end{aligned}
$$

containing natural modes $\Phi$ and frequencies $\Omega$, and also expressions for nodal measurements $C_{m z}, C_{m v}, D_{o a, f b}$

$$
\begin{aligned}
& A=\left[\begin{array}{cc}
0_{(n-2) \times(n-2)} & I_{n-2} \\
-\Omega^{2} & -2 Z \Omega
\end{array}\right] \\
& B=\left[\begin{array}{c}
0_{(n-2) \times n} \\
\Phi^{T}
\end{array}\right] \\
& C=\left[\begin{array}{ll}
C_{m z} & C_{m v}
\end{array}\right] \\
& D=\left[\begin{array}{l}
0_{(n-2) \times n} \\
0_{n \times n} \\
D_{o a, f b}
\end{array}\right] \text {. }
\end{aligned}
$$

Here, $x$ is a $2(n-2) \times 1$ state vector and $u=F=$ $\left(F_{1}, \ldots, F_{n}\right)$ is a $n \times 1$ input vector whilst $y=\left(\varepsilon, q, a_{z}\right)$ is a $(3 n-2) \times 1$ output vector, all for $n=100$ nodes.

The reduced model replaces the $2(n-2) \times 1$ state vector $x$ in (1)-(2) above with only ten states corresponding to the first five modes because they dominate the structure dynamics. That reduced model still has the original number of nodal inputs $u$ and outputs $y$ shown in (1) (2) above. The focus of Sections 3,4 below is optimal determination of the number and location of the smallest possible set of actuators and sensors which can generate the necessary control actions and provide informative feedback measurements [8].

\section{Actuator placement}

Since placing additional actuators on the existing missile airframe is not practical, the already-available actuators (thrust vectoring and the fins) are only considered. The position of these two actuators on the airframe are $x_{n}=0 \mathrm{~m}$ and $x_{f}=2.4 \mathrm{~m}$, corresponding to nodes 1 and 50. It is assumed that the vibrations are mainly excited by lateral forces applied at these two locations. The rear location corresponds to engine thrust unsteady misalignment and the middle one is due to unsteady lift on the fins created by turbulence. The aerodynamic forces along the body are too distributed and too weak to create significant bending when compared to the rocket motor lateral thrust at the rear of the booster. Hence, the only inputs for active vibration damping are lateral forces at nodes 1 , i.e. $F_{1}$, and 50 , i.e. $F_{50}$, see Figure 2. Thus, only columns 1 and 50 of $B$ and $D$ in (1)-2) are retained resulting in the $2(n-2) \times 2$ matrix $B^{*}$ and $(3 n-2) \times 2$ matrix $D^{*}$. 


\section{Sensor placement}

The output vector $y=\left(\varepsilon, q, a_{z}\right)$ of the state-space model (1)-(2) expresses nodal measurements for three different types of possible sensors: $n-2$ strain gauges expressed in $\varepsilon, n$ gyroscopes expressed in $q$ and $n$ accelerometers expressed in $a_{z}$. Given that only the first five natural modes are needed, a much smaller number of feedback measurements is necessary for active vibration damping control. That number of sensors is computed in this section and the optimal locations for the sensors determined using the $H_{\infty}$-norm approach [9, Chapter 7]. More advanced techniques, e.g. [10], are possible but here they were neither necessary nor naturally suited for vibration damping control design.

The key idea of optimal sensor placement for linear time-invariant systems is to maximise the observability of the resulting control system $[8]$ and hence a natural setting for this problem is maximisation of the transfer function response [9, Chapter 7]. Having chosen $F_{1}$ and $F_{50}$ in Section 3 as outputs, the corresponding transfer function is given by

$$
G(s)=C(j s-A)^{-1} B^{*}+D^{*}
$$

and is a $(3 n-2) \times 2$ matrix with $B^{*}$ and $D^{*}$ obtained by retaining only columns 1 and 50 of $B$ and $D$ in (1)-(2).

From the three standard transfer function norms in [9. Chapter 5] as maximisation criteria, the $H_{\infty}$ norm is chosen as the most demanding measure which is also compatible with robust control design. For any inputoutput pair, the corresponding $H_{\infty}$ norm is the peak of the transfer function magnitude over the bandwidth considered and also an upper bound on the output error.

The sensors will be placed considering only the first bending mode whose active damping will be the focus of Section 5. For the ASTER 30 missile, the dominant modes higher than one have natural frequencies above $50 \mathrm{~Hz}$ and are thus above the available actuation bandwidth. The focus on the first bending mode is also due to its light damping together with its parasitic influence on the measurements made by the missile's existing inertial sensors and the seeker. In the absence of a notch filter, the parasitic measurements of the first-mode vibrations can be misinterpreted by these sensors as rigid-body deflections from the desired intercept trajectory.

\subsection{Placement indices}

The actual inputs $\left(F_{1}\right.$ and $\left.F_{50}\right)$ were defined by the actuators already placed on the missile airframe, see Section 3 . Additional sensors for active vibration damping are yet to be placed at locations chosen from all

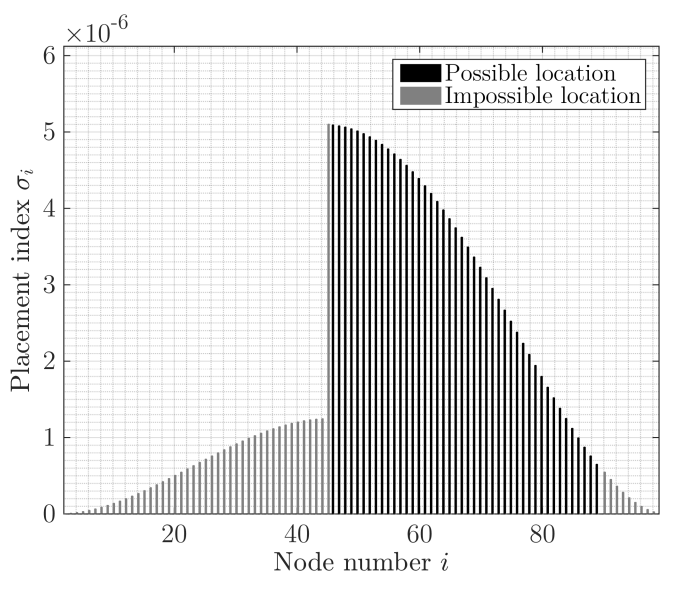

Figure 1: Placement indices for strain gauges

nodes or, equivalently, from all potential outputs. The essence of optimal sensor placement is to compare responses of all input-output pairs in order to choose the actual outputs as a compromise between the response levels and physical accessibility of the chosen nodes.

Let $S=\left\{i_{1}, i_{2}, \ldots, i_{s}\right\}$ be the set of $s$ possible (accessible) locations, chosen from $n=100$ nodes. At each $i \in S$, the $H_{\infty}$-norm index

$$
\sigma_{i}=\left\|G_{1 i}\right\|_{\infty}
$$

is calculated, where $G_{1 i}$ is the transfer function expressing the response level of the sensor to the first mode at $i$ with respect to inputs $F_{1}$ and $F_{50}$.

For the strain gauges and gyroscopes

$$
\left\|G_{1 i}\right\|_{\infty} \approx \frac{\left\|B_{1}\right\|_{2}\left\|C_{i 1}\right\|_{2}}{2 \zeta_{1} \omega_{1}}
$$

because the damping ratio of the first bending mode is only $1 \%$, see [9, Section 7.2.2]. Here, $B_{1}$ and $C_{i 1}$ are the matrices for the first bending mode at node $i$ for the sensor considered. $B_{1}$ is the first two rows of $B$ and $C_{i 1}$ is the first two columns of the part of $C$ corresponding to the type of sensor considered.

For the accelerometers

$$
\left\|G_{1 i}\right\|_{\infty} \approx \omega_{1} \frac{\left\|B_{1}\right\|_{2}\left\|C_{i 1}\right\|_{2}}{2 \zeta_{1} \omega_{1}}=\frac{\left\|B_{1}\right\|_{2}\left\|C_{i 1}\right\|_{2}}{2 \zeta_{1}}
$$

because the lateral acceleration of a node is the derivative of its lateral speed. Here, $B_{1}$ and $C_{i 1}$ are the input and output matrix for the first bending mode with the output being the lateral speed at node $i$.

\subsection{Strain gauges placement}

A strain gauge is a surface-mounted resistor, measuring only the surface deformation so it does not register 
the rigid-body motions. For the ASTER 30 missile, possible (accessible) locations are: $2.2 \mathrm{~m} \leqslant x \leqslant 4.4 \mathrm{~m}$, corresponding to the set of nodes $S_{1}=\{46, \ldots, 89\}$.

Using (9), the placement indices for strain gauges were computed for all locations for comparison with possible locations, see Figure 1. The placement indices at locations on the booster are very low compared to those on the dart because the booster is more stiff than the dart. The best strain gauge location is node 46 at $x=2.23 \mathrm{~m}$ behind the fins, as shown in Figure 2. This location corresponds to the strain anti-node of the first bending mode where the flexure is at a maximum. Similar figures are also used for the gyroscope and the accelerometer placement.

\subsection{Gyroscopes placement}

For a flexible missile airframe, a gyroscope registers at a node $i$ not only the pitch rate due to the vibrations $q_{i, f b}$ but also the pitch rate of the rigid-body $q$ so that

$$
q_{i}=q_{i, f b}+q
$$

is the overall measurement. In order to isolate the vibrations pitch rate, measurements of two gyroscopes placed at different locations $i$ and $j$ must be made. Then, the rigid-body pitch rate can be eliminated:

$$
q_{i}-q_{j}=q_{i, f b}-q_{j, f b} .
$$

For the gyroscopes, the possible locations are: $0.45 \mathrm{~m} \leqslant x \leqslant 4.40 \mathrm{~m}$, corresponding to the set of nodes $S_{2}=\{10, \ldots, 92\}$.

The nose is a place where a gyroscope would be very sensitive to the first bending mode. In the current version of ASTER 30, there already is a gyroscope close to node 83 . Another gyroscope must be placed as per (12) with two best positions being node 83 and node 10 .

\subsection{Accelerometer placement}

Accelerometers measure the lateral acceleration due to vibrations but also the lateral acceleration of the rigidbody. At node $i$, an accelerometer measures:

$$
a_{z, i}=a_{z, r b, C G}+\left(x_{C G}-x_{i}\right) \dot{q}+a_{z, f b, i}
$$

so there are three unknowns: $a_{z, r b, C G}, \dot{q}$ and $a_{z, f b, i}$. Hence, at least three accelerometers are needed at different locations to eliminate the acceleration of the centre of gravity 1 and the term due to pitch acceleration.

For the accelerometers, the possible locations are: $0.45 \mathrm{~m} \leqslant x \leqslant 4.40 \mathrm{~m}$, corresponding to the set of nodes $S_{3}=\{10, \ldots, 92\}$.

\footnotetext{
${ }^{1}$ It is shown in Section 5.3 that the exact location of the centre of gravity is not needed to isolate the acceleration due to vibrations.
}

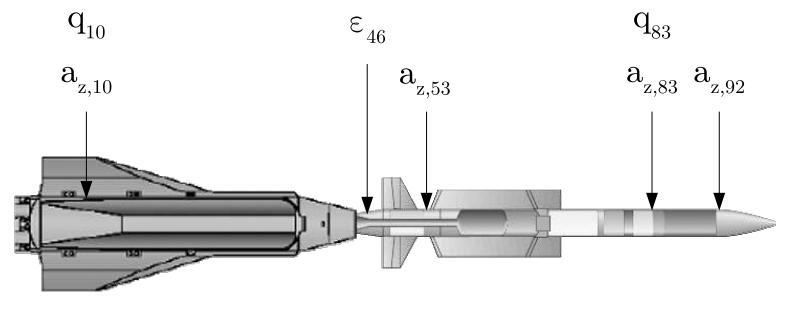

Figure 2: Output selection: strain gauges $\varepsilon$, gyroscopes $q$ and accelerometers $a_{z}$; missile length is $4.9 \mathrm{~m}$ and there are $n=100$ uniformly-spaced nodes as candidate locations for sensor placement

In the current version of ASTER 30, there already is an accelerometer close to node 83. This location is not very sensitive to the lateral accelerations due to first bending mode so this existing accelerometer cannot be used for bending vibration control. The three best locations at nodes 10, 53 and 92, see Figure 2

\subsection{Output selection}

The results derived in Sections 4.2 4.4 lead to the following output selection: (i) existing gyroscope and accelerometer at node 83 , (ii) additional strain gauge at node 46 , (iii) additional gyroscope at node 10 , (iv) additional accelerometers at nodes 10, 53 and 92, as illustrated in Figure 2 .

\subsection{Aeroservoelastic model: Inputs and outputs}

Based on Sections 3 and 4.5, the aeroservoelastic model has the following inputs and outputs. Reference acceleration This is a desired input resulting from the guidance demand. Control inputs These are available inputs for the guidance demand and vibration damping: the thrust vectoring orientation and fins deflection. Perturbation inputs These are unwanted inputs: sensor noise and perturbation forces. Sensor outputs These are measurements from strain gauges, gyroscopes and accelerometers. The accelerometers and gyroscopes measure both the rigid-body the flexible-body effects which can be separated, see (11)- 13. Performance outputs These are auxiliary outputs which are not measured but can be used to assess controller performance.

The corresponding Simulink diagram of the aeroservoelastic model is shown in Figure 3

\section{Active vibration damping control}

For ASTER 30, the natural frequency of the first bending mode is within the actuation bandwidth. The airframe deformation corresponding to that mode may be misinterpreted by the autopilot as deviation from 


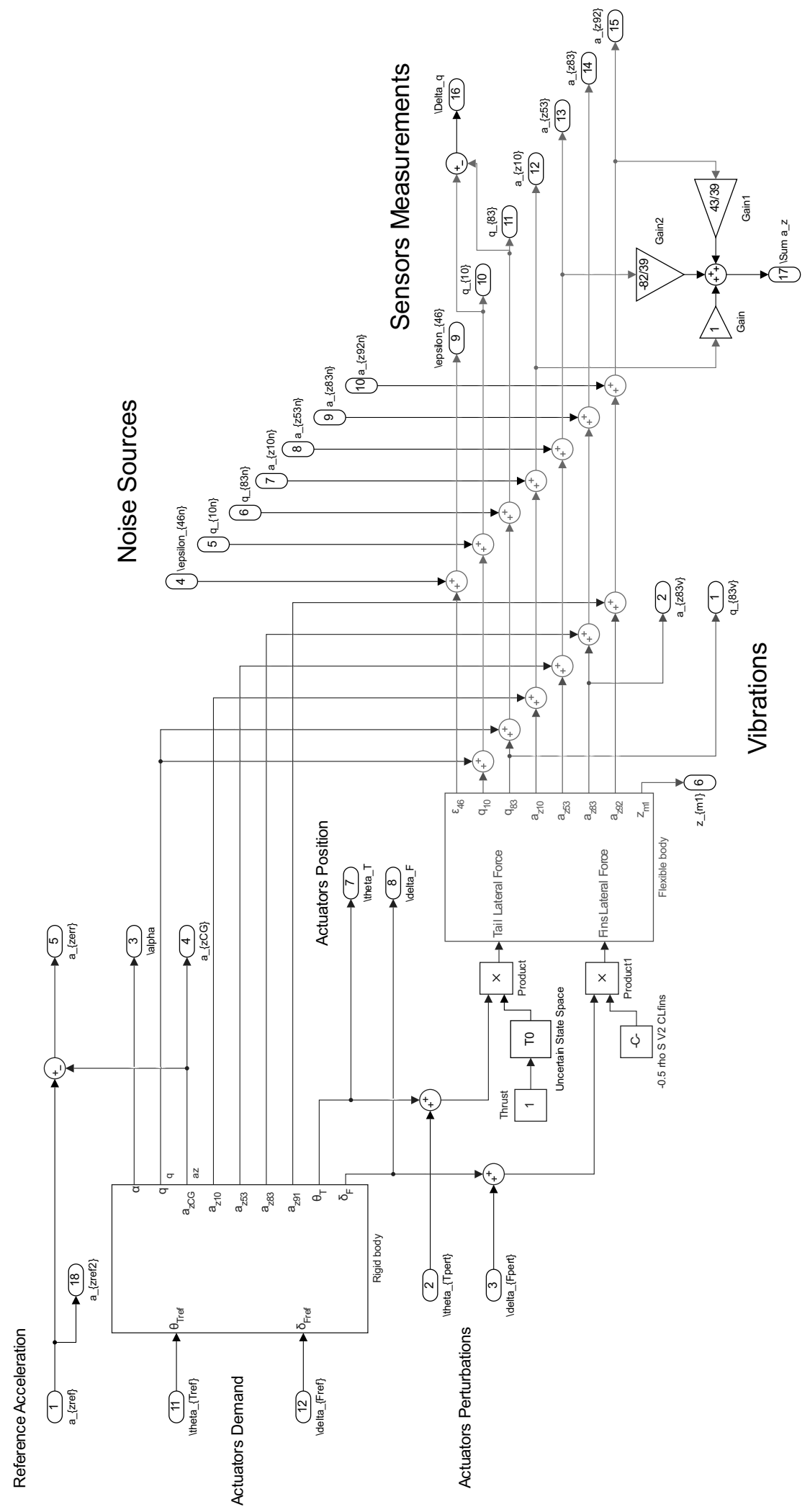

Figure 3: Inputs and outputs for the aeroservoelastic model, see Section 4.6 
desired rigid-body trajectory, potentially leading to instability. Instead of the standard solution employing a notch filter, the focus here is to counter bending oscillations by using active vibration damping. Three variants of closed-loop active vibration damping control are considered: 1) strain gauge feedback in Section 5.1. 2) gyroscope feedback in Section 5.2, and 3) accelerometer feedback in Section 5.3

Active vibration damping controller must generate a force that is opposite to the vibration rate of the first mode whose natural frequency is $20 \mathrm{~Hz}$. The bandwidth of the thrust vectoring is about $25 \mathrm{~Hz}$ which is too low for such force generation and the real behaviour of the actuator so close to the cut-off frequency is not accurately modelled. On the other hand, the fins have a bandwidth of $50 \mathrm{~Hz}$ with the phase loss at $20 \mathrm{~Hz}$ of only $-34^{\circ}$ and the gain loss of $-0.1 \mathrm{~dB}$. Hence, the fin actuation is to be considered for active damping of the first bending mode, especially that the fins are located where the flexure is significant so that good controllability is assured.

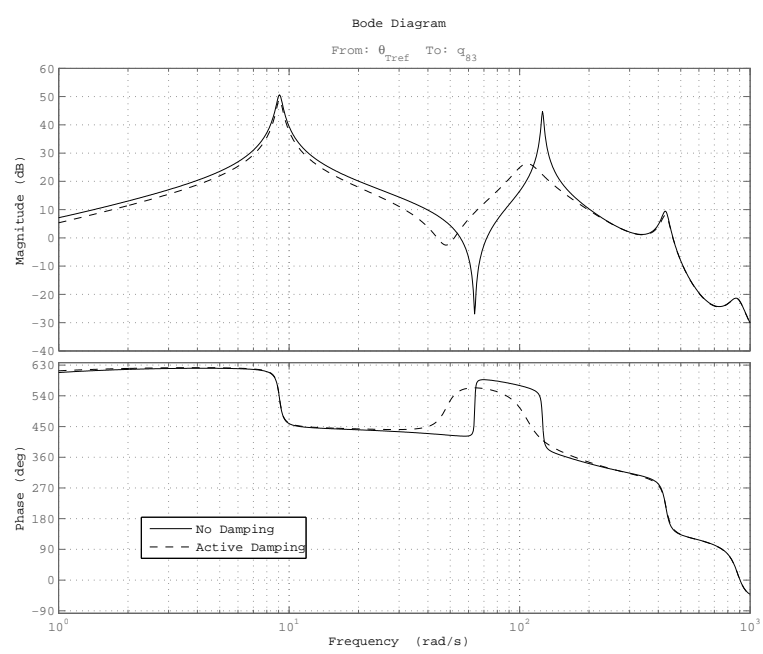

Figure 4: The Bode plot of $q_{83} / \theta_{T}^{\mathrm{ref}}(s)$ with and without strain feedback, see Figure 12 a

\subsection{Strain feedback}

An optimal placement for a strain gauge is node 46 (see Section 4.2). The corresponding transfer function from the fins deflections to the strain $\varepsilon_{46} / \delta_{F}(s)$ is:

$$
K_{\frac{\varepsilon_{4}}{\delta_{F}}} \frac{\omega_{1}^{2}}{s^{2}+2 \zeta_{1} \omega_{1} s+\omega_{1}^{2}},
$$

where $K_{\frac{\varepsilon_{46}}{\delta_{F}}}$ is the static gain.
In order to increase the damping term $\zeta_{1}$ in $(14)$, the derivative of the strain measurement is fed back, resulting in the closed-loop transfer function

$$
K \frac{\varepsilon_{46}}{\delta_{F}} \frac{\omega_{1}^{2}}{s^{2}+2\left(\zeta_{1}+\frac{1}{2} K_{\frac{\varepsilon_{46}}{\delta_{F}}} \omega_{1} K_{\varepsilon}\right) \omega_{1} s+\omega_{1}^{2}} .
$$

The feedback gain $K_{\varepsilon}$ changes the damping ratio of the first mode without changing the static gain or natural frequency $\omega_{1}$. Since pure derivative feedback $K_{\varepsilon} s$ is non-causal, a fast-pole implementation $K_{\varepsilon} s /\left(1+T_{\varepsilon} s\right)$ is used with $T_{\varepsilon}$ suitably small.

With a proportional feedback gain of 600 , the damping ratio of the first bending mode is $12.5 \%$, resulting in a gain margin of $6 \mathrm{~dB}$ and a phase margin of $30^{\circ}$. Figure 4 of the Bode diagram for $q_{83} / \theta_{T}^{\text {ref }}(s)$ clearly shows that the first bending mode is well damped. The resonance peak at 125 rad.s ${ }^{-1}$ is reduced by $20 \mathrm{~dB}$.

Figure 12p shows the complete feedback system with the strain feedback, the pitch rate loop and PI autopilot.

\subsection{Gyroscope feedback}

As discussed in Section 4.3. optimal placement of two gyroscopes can give flexure measurement:

$$
q_{10}-q_{83}=q_{f b, 10}-q_{f b, 83}=\Delta q .
$$

The transfer function of the fins deflection $\delta_{F}$ to the pitch rate difference $\Delta q$ is

$$
K_{\frac{\Delta q}{\delta_{F}}} \frac{\omega_{1}^{2} s}{s^{2}+2 \zeta_{1} \omega_{1} s+\omega_{1}^{2}} .
$$

Applying proportional feedback gain $K_{\Delta q}$ results in the closed-loop transfer function:

$$
K_{\frac{\Delta q}{\delta_{F}}} \frac{\omega_{1}^{2} s}{s^{2}+2\left(\zeta_{1}+\frac{1}{2} K_{\frac{\Delta q}{\delta_{F}}} \omega_{1} K_{\Delta q}\right) \omega_{1} s+\omega_{1}^{2}} .
$$

A damping of $12 \%$ on the first bending mode is achieved with a feedback gain $K_{\Delta q}=0.24$. The resonance peak of the first bending mode is reduced by $20 \mathrm{~dB}$ is shown in Figure 5. A diagram of the complete feedback system is shown in Figure 12 .

\subsection{Accelerometer feedback}

As explained in Section 4.4, three accelerometers are added at nodes 10, 53 and 92 because three uncorrelated accelerometers are needed to isolate the flexible body component. A linear combination of these three measurements can be found so that it does not depend on $a_{z, C G}$ and $\dot{q}$. Considering only the first bending mode 


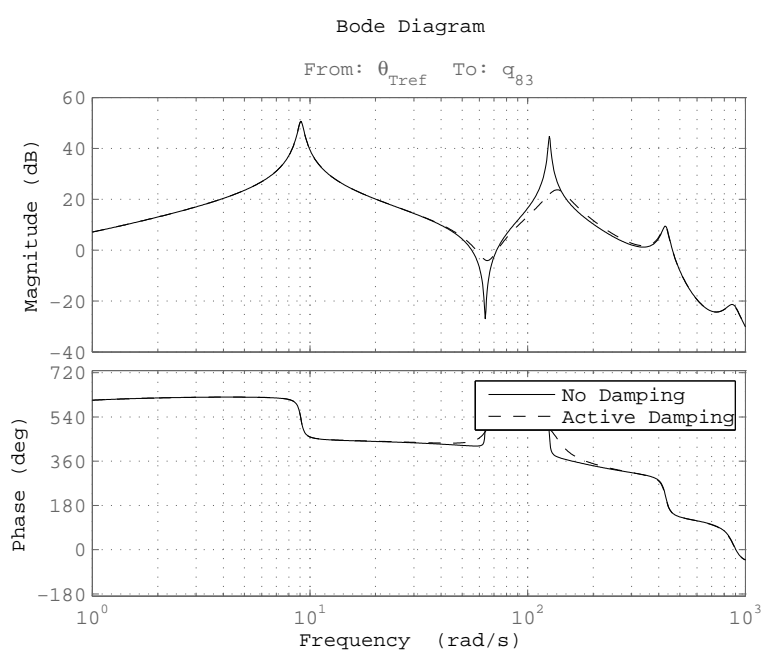

Figure 5: The Bode plot of $q_{83} / \theta_{T}^{\text {ref }}(s)$ with and without strain feedback, see Figure 12 p

at nodes $i=10,53,92$, the corresponding $a_{z i, f b}$ are proportional to the mean acceleration $a_{z, m 1}$.

Let $c_{10}, c_{53}, c_{92}$ be three coefficients satisfying

$$
c_{10} a_{z, 10}+c_{53} a_{z, 53}+c_{92} a_{z, 92}=a_{z, m 1}
$$

so that applying (19) repeatedly leads to

$$
\left[\begin{array}{l}
c_{10} \\
c_{53} \\
c_{92}
\end{array}\right]=\left[\begin{array}{r}
1 \\
-\frac{82}{39} \\
\frac{43}{39}
\end{array}\right] .
$$

Let $\sum a_{z}$ denote " $c_{10} a_{z, 10}+c_{53} a_{z, 53}+c_{92} a_{z, 92}$ " for brevity. The transfer function of the fins deflection input to the linear combination of accelerations output $\sum a_{z} / \delta_{F}(s)$ is:

$$
K_{\frac{\sum_{a_{z}}}{\delta_{F}}} \frac{\omega_{1}^{2} s^{2}}{s^{2}+2 \zeta_{1} \omega_{1} s+\omega_{1}^{2}} .
$$

In order to actively damp the first bending mode, integral feedback is applied with the resulting closed-loop transfer function:

$$
K_{\frac{\sum_{a_{z}}}{\delta_{F}}} \frac{\omega_{1}^{2} s^{2}}{s^{2}+2\left(\zeta_{1}+K_{\frac{\sum_{a_{z}}}{\delta_{F}}} \omega_{1} K_{a_{z}}\right) \omega_{1} s+\omega_{1}^{2}} .
$$

The first bending mode is damped $12 \%$ with a gain $K_{a_{z}}=0.16$. The corresponding Bode plot in Figure 6 shows vibration damping effect on the resonance peak. Finally, the complete feedback system is shown in Figure 12.

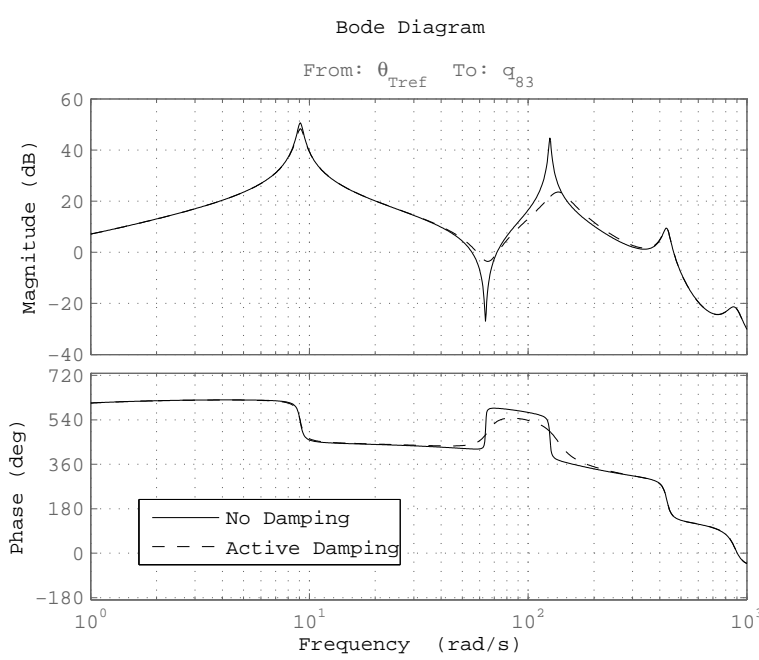

Figure 6: The Bode plot of $q_{83} / \theta_{T}^{\text {ref }}(s)$ with and without $\sum a_{z}$ feedback, see Figure 12 .

\subsection{Robust tuning of controllers}

Three active vibration damping controllers shown in Figure 12 were tuned; for comparison a notch-filter controller (without active vibration damping) was also considered, and the results are marked "Notch", "Strain", "Gyro" and "Acc". Apkarian's $H_{\infty}$-tuning for fixedstructure controllers [11] was used in the weighted form. The input to the closed-loop system is the exogenous vector $w$ which contains all the inputs, see Figures 3 and 7, whilst the output is the performance vector $z$. The diagonal matrices $W_{\text {in }}$ and $W_{\text {out }}$ are weights applied to $w$ and $z$ to define the requirements.

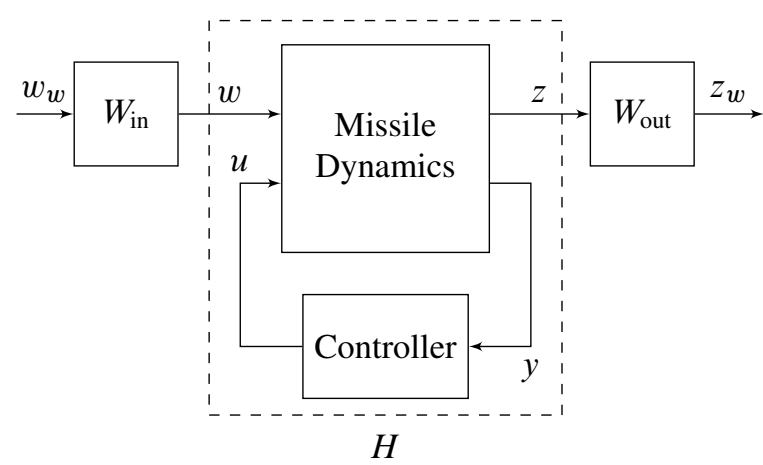

Figure 7: Weighted form for $H_{\infty}$-tuning

The controller gains $K_{q}, K_{P I}$ and $\tau_{P I}$, see Figure 12 , will be tuned in order to make the system stable while minimising $\gamma$ such that

$$
\left\|W_{\text {out }} H W_{\text {in }}\right\|_{\infty}<\gamma .
$$


The exogenous vector $w$ only contains the reference lateral acceleration $a_{z}^{\text {ref }}$. The corresponding weight is set to 1 so the other weights are chosen with respect to a reference acceleration of $1 \mathrm{~m} \cdot \mathrm{s}^{-1}$. Sensor noise is low compared to signals generated by vibrations and the thrust vectoring perturbations dominate.

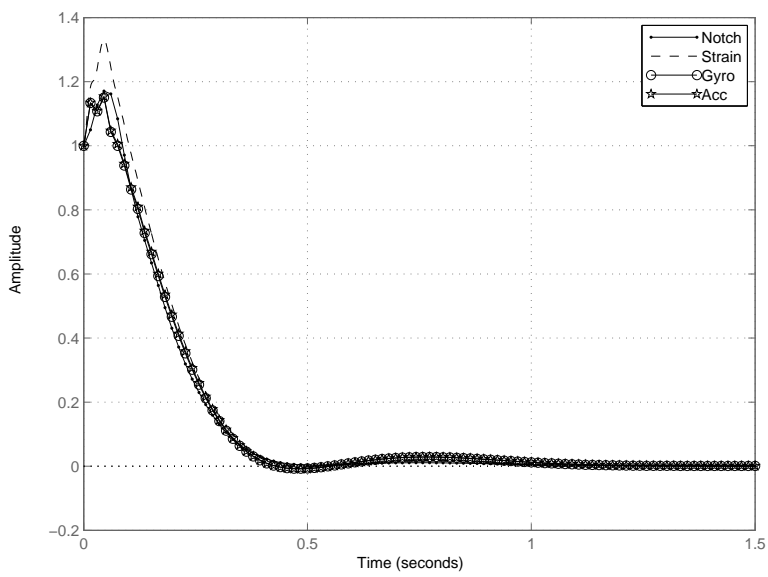

Figure 8: Step response of $a_{z}^{\text {ref }}$ to $\left(a_{z}^{\text {ref }}-a_{z C G}\right)$

The performance vector $z$ is composed of two signals: the lateral acceleration error $a_{z}^{\mathrm{ref}}-a_{z C G}$ and the thrust vectoring orientation $\theta_{T}$. The thrust vectoring orientation $\theta_{T}$ is added in $z$ to limit the use of this actuator which has a rate limit, a deflection limit and secondorder dynamics. The output weight matrix $W_{\text {out }}$ is diagonal with elements $W_{\text {err }}$ and $W_{\theta_{T}}$. The empirical value of $W_{\text {err }}$ minimising the error at low frequencies is:

$$
W_{\text {err }}(s)=\frac{5}{s}\left(\frac{s}{10}+1\right)
$$

which produces the bandwidth of $5 \mathrm{rad} / \mathrm{s}$. Finally, $W_{\theta_{T}}$ is chosen to avoid actuation above the cutoff frequency:

$$
W_{\theta_{T}}(s)=2 \cdot 10^{-3}\left(\frac{s}{\omega_{T}}\right)^{2}\left(\frac{1}{1+10^{-5} s}\right)^{2},
$$

where $\omega_{T}$ is the thrust vectoring cutoff frequency of $25 \mathrm{~Hz}(157 \mathrm{rad} / \mathrm{s})$; the coefficient $2 \cdot 10^{-3}$ is empirical.

\begin{tabular}{|c|c|c|c|}
\hline Controller & $K_{q}[\mathrm{~s}]$ & $K_{P I}[\mathrm{rad} \cdot \mathrm{s} / \mathrm{m}]$ & $\tau_{P I}[\mathrm{~s}]$ \\
\hline \hline Notch & $9.47 \cdot 10^{-2}$ & $9.46 \cdot 10^{-3}$ & 0.171 \\
\hline Strain & $9.98 \cdot 10^{-2}$ & $13.0 \cdot 10^{-3}$ & 0.122 \\
\hline Gyro & $8.40 \cdot 10^{-2}$ & $9.11 \cdot 10^{-3}$ & 0.149 \\
\hline Acc & $8.38 \cdot 10^{-2}$ & $9.05 \cdot 10^{-3}$ & 0.151 \\
\hline
\end{tabular}

Table 1: Parameters of $\mathrm{H}_{\infty}$-tuned controllers
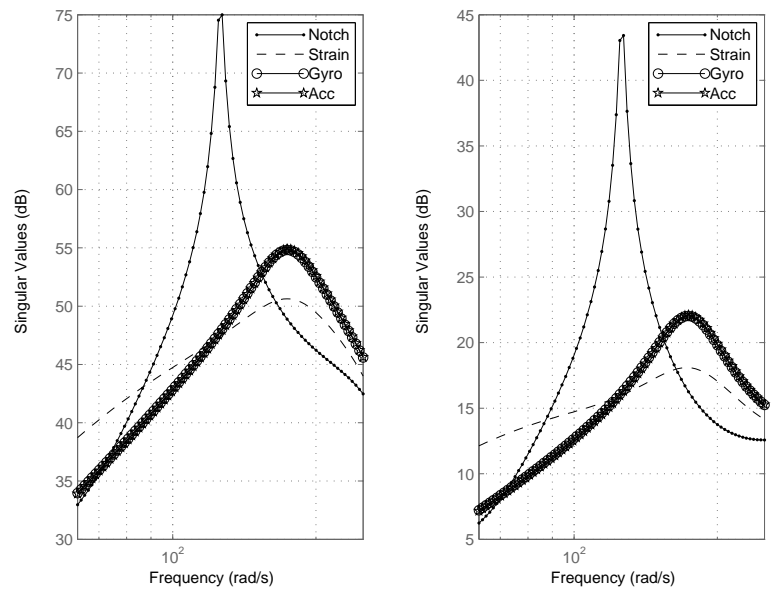

Figure 9: Singular values of $\theta_{T}^{\text {pert }}$ to $a_{z 83 v}$ (left) and $q_{83 v}$ (right)

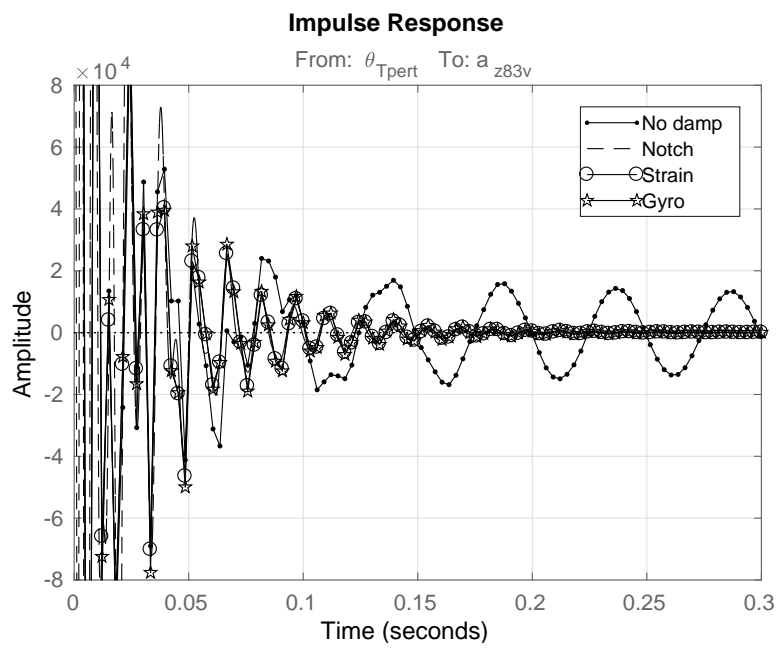

Figure 10: Impulse response of $\theta_{T}^{\text {pert }}$ to $a_{z 83 v}$

The $\mathrm{H}_{\infty}$-tuning yields parameters are summarised in Table 1. The "Notch" controller suppresses vibrationinduced noise with the notch filter without removing the vibrations whilst the active damping controllers "Strain", "Gyro" and "Acc" suppress the vibrations directly. The tracking performance of these four closedloop controllers is illustrated by their step responses, see Figure 8 , showing that all approaches give similar results: the rise time from 10 to $90 \%$ following a step demand is between 0.214 and 0.227 seconds and the $2 \%$ settling time is between 0.400 and 0.406 seconds.

However, the active damping controllers have the advantage of reducing bending vibrations generated by the rocket engine and aerodynamic turbulence. The corresponding singular values are shown in Figure 9 where $\theta_{T}^{\text {pert }}$ is the noisy thrust deflection whilst $a_{z 83 v}$ and $q_{83 v}$ 


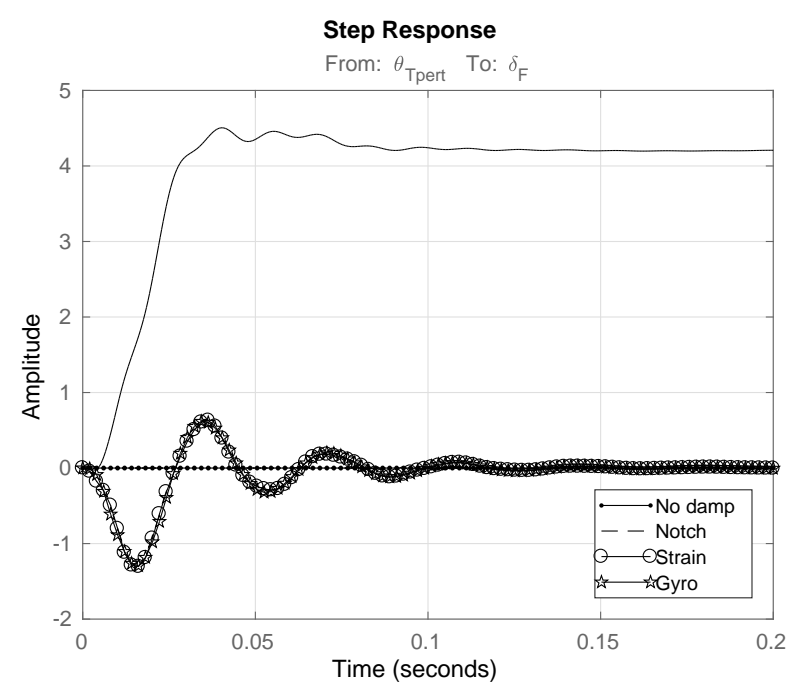

Figure 11: Step response of $\theta_{T}^{\text {pert }}$ to $\delta_{F}$

are the vibration component of acceleration and pitch rate at the location of existing ASTER 30 sensors. It can be seen that the "Notch" design does nothing to reduce vibrations whereas the other three reduce them considerably. Figure 10, shows that after an impulse of the thrust orientation, the "Notch" results in a residual low frequency vibration whereas the other architectures damp it quickly.

From the fins actuation demand point of view, Figure 11 shows the fins deflection $\delta_{F}$ controlled by each loop due to a noisy thrust deflection step $\theta_{T}^{\text {pert }}$. It should be noted that the "Notch" design does not control this actuator. The "Acc" and "Gyro" designs have a transient state where the first bending mode is being damped. The "Strain" design has the disadvantage of having a non-zero static gain, thus a static bend results in a static fins deflection.

\section{Conclusions}

An aeroservoelastic model of a slender anti-air missile was developed by discretising continuous EulerBernoulli beam, reducing the model to the first five bending modes because they dominate vibration generation. A small number of additional sensors (strain gauges, gyroscopes and accelerometers) was optimally placed on the missile airframe to maximise the first mode observability. This optimal sensor placement allowed design of three new autopilots for active damping control of the first mode using the missile's middle fins as the actuator. These active damping controllers were optimally tuned using Apkarian's $H_{\infty}$ structured synthesis. Compared to the traditional autopilot design, which uses a bandwidth-limiting notch filter to restrict the influence of the first structural mode, the new autopilots demonstrated effective oscillation damping. This effective damping reduced vibrations whilst produced low actuation demand, thus enabling better missile performance.

\section{Acknowledgments}

The authors thank MBDA for suggesting the topic of this work and for providing relevant advice.

\section{References}

[1] F. W. Nesline Jr, M. L. Nesline, Phase vs. gain stabilization of structural feedback oscillations in homing missile autopilots, in: 1985 American Control Conference, IEEE, 1985, pp. 323-329.

[2] D. H. Platus, Aeroelastic stability of slender, spinning missiles, Journal of Guidance, Control, and Dynamics 15 (1) (1992) 144151.

[3] C. H. Murphy, W. H. Mermagen, Flight mechanics of an elastic symmetric missile, Journal of Guidance, Control, and Dynamics 24 (6) (2001) 1125-1132.

[4] H. Haddadpour, Aeroservoelastic stability of supersonic slender-body flight vehicles, Journal of Guidance, Control, and Dynamics 29 (6) (2006) 1423-1427.

[5] M. Ehramianpour, H. Haddadpour, M. T. Ahmadian, Aeroelastic behavior of a slender body considering free fittings, Journal of Mechanical Science and Technology 24 (9) (2010) 1755-1762.

[6] D. Lesieutre, J. Love, M. Dillenius, A. Blair Jr, Recent applications and improvements to the engineering-level aerodynamic prediction software misl3, in: Proceedings of the 40th AIAA Aerospace Sciences Meeting and Exhibit, 2002, pp. 2002-0275.

[7] J. M. Prentis, F. A. Leckie, Mechanical vibrations: An introduction to matrix methods, Longmans, London, 1963.

[8] M. van de Wal, B. de Jager, A review of methods for input/output selection, Automatica 37 (4) (2001) 487-510.

[9] W. K. Gawronski, Advanced Structural Dynamics and Active Control of Structures, Springer, New York, 2004.

[10] B. B. Li, A. D. Kiureghian, Robust optimal sensor placement for operational modal analysis based on maximum expected utility, Mechanical Systems and Signal Processing 75 (15 June) (2016) 155-175.

[11] P. Apkarian, D. Noll, Nonsmooth $\mathrm{H}_{\infty}$ synthesis, IEEE Transactions on Automatic Control 51 (1) (2006) 71-86, [Erratum in 2006, 51 (2), 382]. 


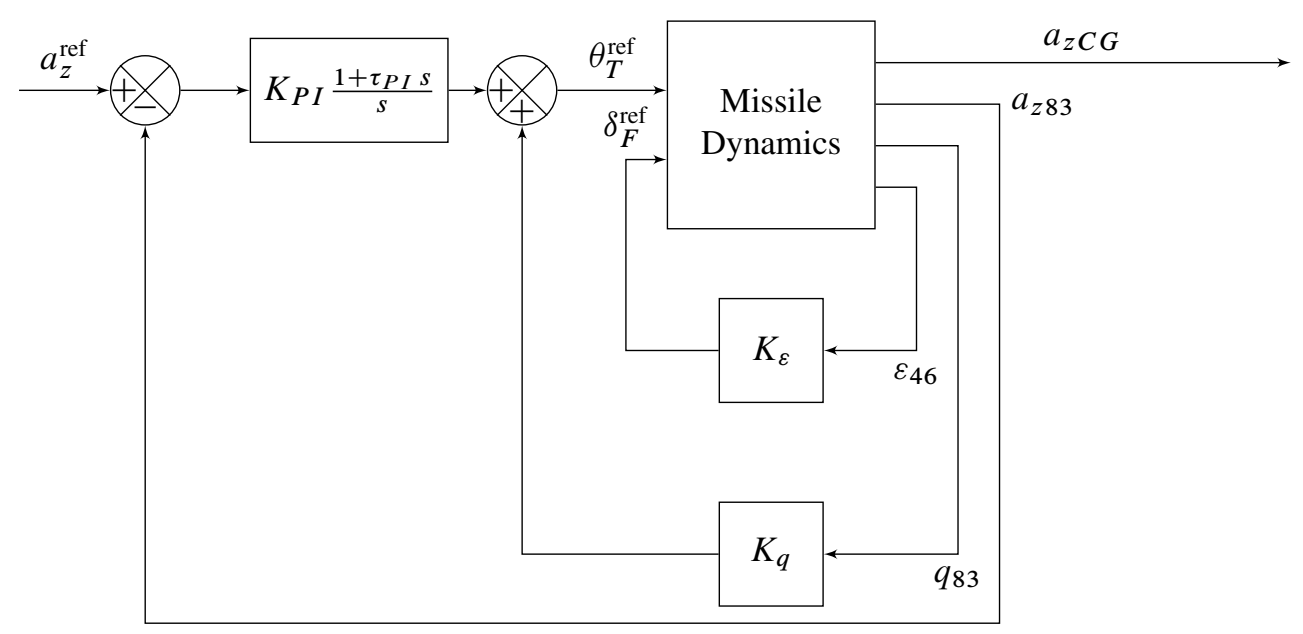

a)

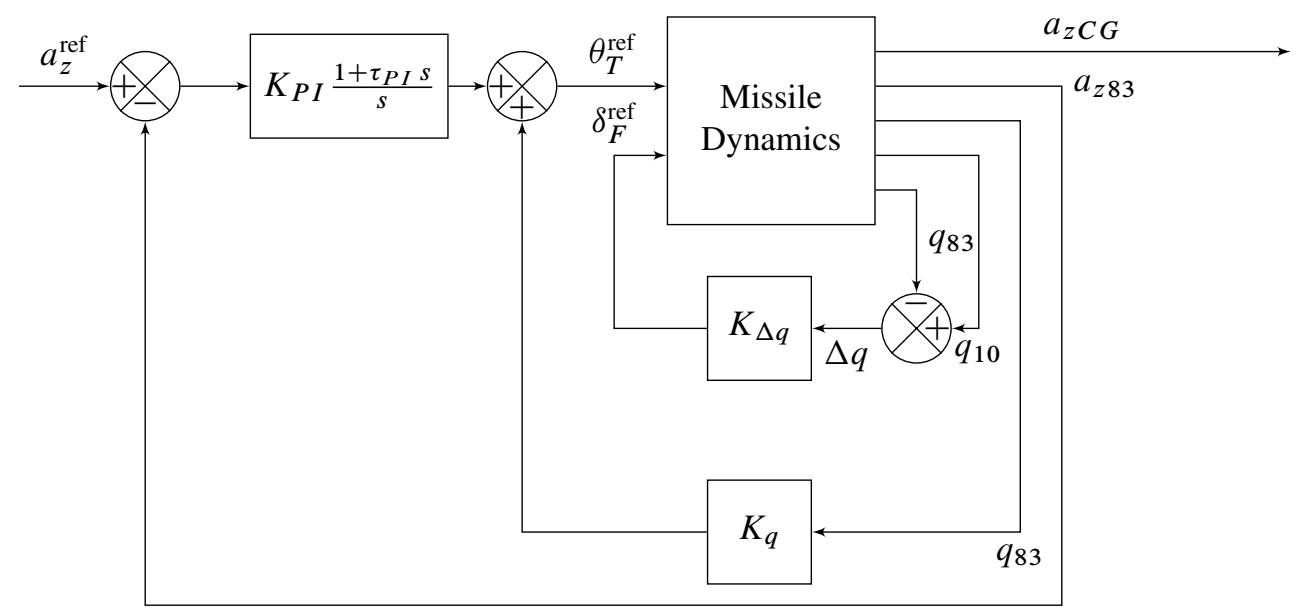

b)

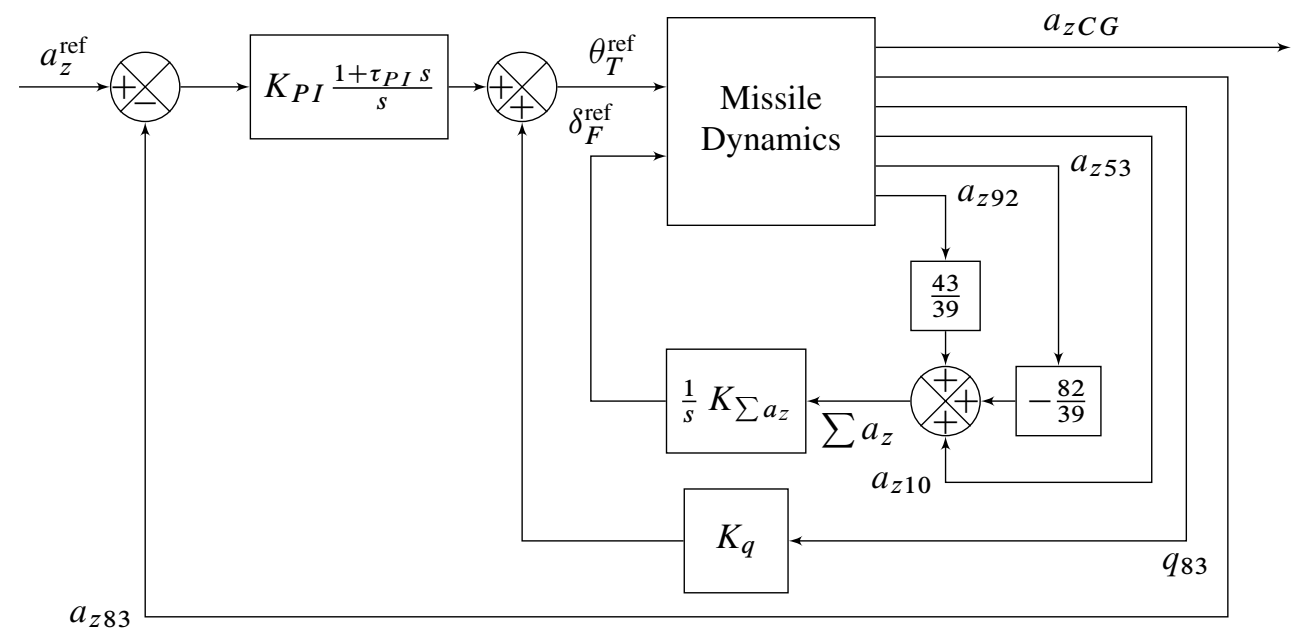

c)

Figure 12: Three variants of closed-loop active vibration damping control: a) strain gauge feedback, see Section 5.1 b) gyroscope feedback, see Section 5.2 c) accelerometer feedback, see Section 5.3 Note the presence of feedback from the existing ASTER 30 gyroscope $q_{83}$ in all variants 


\title{
Aeroservoelastic modelling and control of a slender anti-air missile for active damping of longitudinal bending vibrations
}

\author{
Verhaegen, Anatole
}

Elsevier

pÿAnatole Verhaegen, RafaB \{bikowski, Aeroservoelastic modelling and control of a slender anti-air missile for active damping of longitudinal bending vibrations, Aerospace Science and Technology, Volume 66, July 2017, Pages 20-27

http://dx.doi.org/10.1016/j.ast.2017.03.001

Downloaded from Cranfield Library Services E-Repository 